\title{
Investigating the effects of fluidic connection between microbial fuel cells
}

\author{
Jonathan Winfield, Ioannis Ieropoulos, John Greenman and Julian Dennis
}

\begin{abstract}
Microbial fuel cells (MFCs) can 'treat' wastewater but individually are thermodynamically restricted.

Scale-up might, therefore, require a plurality of units operating in a stack which could introduce losses simply through fluidic connections. Experiments were performed on two hydraulically joined MFCs (20 cm apart) where feedstock flowed first through the upstream unit (MFCup) and into the downstream unit (MFCdown) to explore the interactive effect of electrical load connection, influent make-up and flow-rate on electrical outputs. This set-up was also used to investigate how calculating total internal resistance based on a dynamic open circuit voltage (OCV) might differ from using the starting OCV. When fed a highly conductive feedstock $(4,800 \mu \mathrm{S})$ MFCdown dropped approximately $180 \mathrm{mV}$ as progressively heavier loads were applied to MFCup (independent of flow-rate) due to electron leakages through the medium. The conductivities of plain acetate solutions (5 and 20 $\mathrm{mM}$ ) were insufficient to induce losses in MFCdown even when MFCup was operating at high current densities. However, at the highest flow-rate ( $240 \mathrm{~mL} / \mathrm{h})$ MFCdown dropped by approximately $100 \mathrm{mV}$ when using 5 and $220 \mathrm{mV}$ using $20 \mathrm{mM}$ acetate. When the distance between MFCs was reduced by $5 \mathrm{~cm}$, voltage drops were apparent even at lower flow-rates, ( $30 \mathrm{~mL} / \mathrm{h}$ decreased the voltage by $115 \mathrm{mV}$ when using $20 \mathrm{mM}$ acetate). Shear flow-rates can introduce dissolved oxygen and turbulence all capable of affecting the anodic biofilm and redox conditions. Calculating total internal resistance using a dynamic OCV produced a more stable curve over time compared to that based on the starting constant OCV.
\end{abstract}

Keywords Microbial fuel cell; Internal resistance; Wastewater treatment; Open circuit voltage; Stack

\section{Introduction}

The potential for using microbial fuel cells (MFCs) to treat wastewater has long been realised [1], however as yet there are no published accounts of pilot-scale tests carried out at treatment plants [2]. Wastewater treatment requires the removal of organic pollutants and many varieties of waste have successfully been 'treated' by MFCs including swine wastewater [3], brewery wastewater [4] and landfill leachate [5]. However, for MFCs to qualify as a novel technology for the treatment of wastewater, organic content reduction needs to be optimised and electricity production needs to be sufficient to power target applications, a challenge that has proven to be a major obstacle to scale-up since an individual MFC can only generate a working voltage of about $0.5 \mathrm{~V}$ [6]. There have been significant improvements in the power produced by laboratory models over recent years, but invariably environmentally unsound chemicals or expensive materials are used and even the performance of these unsustainable MFCs is not sufficient alone to run useful electrical equipment. With such individual constraints it is perhaps inevitable that scale-up will be achieved using a high number of smaller units rather than a few large ones [7]. To generate enough power these will need to be connected electrically in stacks which will introduce problems not encountered using single MFCs, e.g. cell reversal [8]. These losses will not only affect immediate MFC performance but such phenomena can harm the microbial contingent [9]. Operating a 
plurality of MFCs in an aqueous environment will present the problem of fluidic connection, whether via a common feedline or simply due to the close proximity of individual units. This could lead to further losses that might be in the form of parasitic cross currents taking place between electrically and hydraulically connected MFCs [10] or via ionic shortcut currents produced as ions travel through the conductive media [11].

Microorganisms have always played a major role in the treatment of wastewater, but where exactly MFCs might fit into the process is yet to be realised. Until recently, the majority of MFC research was conducted in batch mode where food/fuel is fed into the anodic chamber and replaced once depleted. The trickling filter is an example of a treatment process which operates in continuous flow rather than batch-mode; wastewater is progressively 'cleaned' in a cascade manner as it seeps through piles of rocks each colonised with complex microbial communities. When taking into account the high volumes and flow-rates operating in real wastewater treatment plants a cascade of miniature MFCs might then be a realistic system for treatment and with the bonus of producing electricity. Such a set-up is likely to require a hydraulic linkage between MFCs and so the plausibility of a MFC cascade system was investigated by looking at the effect that the fluidic connection has on two joined MFCs in relation to electrical load connection, influent make-up and flow-rate. An integral constraint determining the efficiency of a MFC whether operating singularly or in a stack is the internal resistance (RINT). A method ubiquitous in the field of physics for calculating the total RiNT of a cell is based on Kirchoff's voltage law and uses the open circuit voltage (OCV) [7]. A recent proposal has suggested that the OCV might be a dynamic rather than static value [12], a detail that would impact on RINT calculations particularly during longterm monitoring. In relation to this, a method for continuously calculating the OCV of aircraft batteries has been proposed [13], indicating that it is an issue encountered in other fields. To the authors' knowledge this is the first time that such a phenomenon and a proposed method for monitoring are reported for MFCs.

\section{Materials and methods}

\section{MFC construction, inoculation and operation}

Figure 1 shows the basic design of a mini-tubular MFC. A 0.5 x $0.5 \mathrm{~cm} 2$ window was cut towards the bottom of a $1 \mathrm{~mL}$ pipette tip in the narrowing region. Proton exchange membrane (PEM) (VWR, Leicestershire, UK) (2 x $1 \mathrm{~cm}$ ) was adhered over the window using double-sided adhesive (SPI Supplies, West Chester, USA). The anode was constructed from carbon veil (total surface area: $10 \mathrm{~cm}^{2}$ ) folded in half and rolled to create a porous cylindrical 3D structure (projected surface area: $\left.1.96 \mathrm{~cm}^{2}\right)$. A piece of nickel chrome wire $(8 \mathrm{~cm})$ was used to make a connection point with the electrode. The end of the wire was pulled through the narrow end of the pipette tip so that the anode was held firmly against the PEM window. Two pieces of silicon tubing (5 $\mathrm{cm}$ long with $4 \mathrm{~mm}$ internal diameter) were pulled over each end, making a water-tight seal against the pipette tip. A window was cut over the PEM window before the cathode (4 x $10 \mathrm{~cm}$ piece of carbon veil) was wrapped around. It was held in place by double-sided adhesive before a piece of nickel chrome wire $(8 \mathrm{~cm})$ was threaded through. Two pieces of parafilm were wrapped around to hold the cathode firmly against the window and keep the wire secured. A plastic Y-connector was slotted into the bottom tube with the anode wire coming out through one of its channels before aquarium sealant (Wet Water Sticky Stuff, Barry Read Supplies, Ivybridge, UK) was used to seal and block. The tubing at the top acted as the influent entry point and the unsealed Yconnector channel at the bottom was the effluent exit point (see Fig. 1b). 


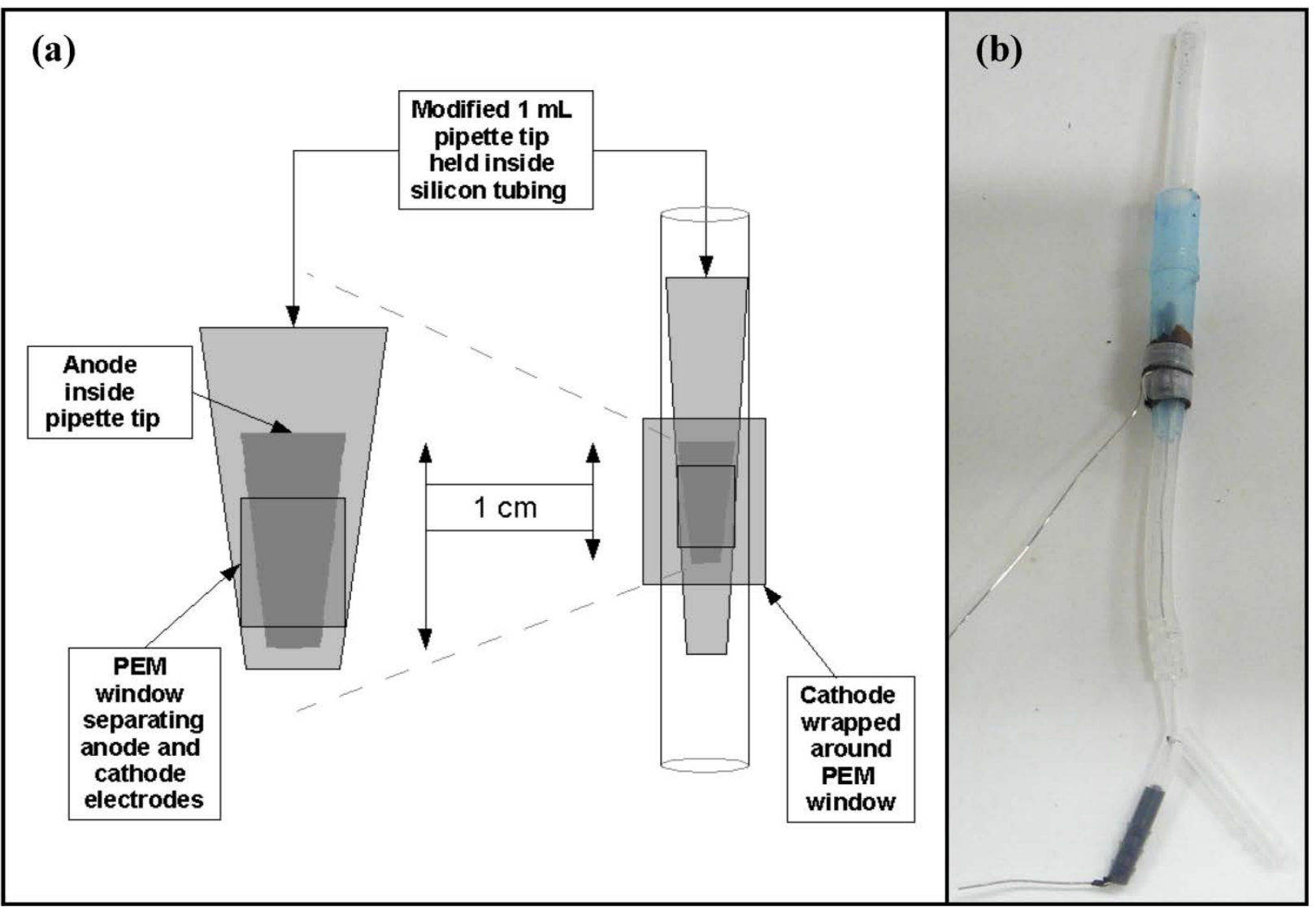

Fig. 1 (a) Schematic representation of a mini-tubular MFC built using $1 \mathrm{~mL}$ pipette tip, silicon tubing and carbon veil as electrode material, (b) photograph of mini-tubular MFC

MFCs were inoculated individually using $1 \mathrm{~L}$ of primary effluent (Wessex Water, Saltford, UK) enriched with $10 \mathrm{~g}$ tryptone and $5 \mathrm{~g}$ yeast extract. For the maturing stage, this was fed at $1.5 \mathrm{~mL} / \mathrm{h}$ in a cyclic fashion using a 16-channel peristaltic pump (Watson Marlow 205U) and the cathode was hydrated with tap water at the same flow-rate. After 3 days running under open circuit conditions, an $8.2 \mathrm{k} \Omega$ load was applied to each MFC. A fresh batch of inoculant was fed after 14 days and by the end of the fourth week the MFCs demonstrated efficient electro-active activity, verified by polarisation experiments. To create a cascade system, MFCs were joined together using silicon tubing ( $4 \mathrm{~mm}$ diameter) and plastic connectors. Figure 2 shows the schematic set-up of the cascade where two MFCs were joined such that the influent flowed through the upstream MFC (MFCup) and into the downstream MFC (MFCdown). The distance between the two MFCs was $20 \mathrm{~cm}$ before being reduced to $15 \mathrm{~cm}$, as part of the distance investigation. For the dynamic open circuit experiments the distance was increased to $40 \mathrm{~cm}$. Three feedstocks were used during experimentation; two concentrations of acetate (5 and $20 \mathrm{mM}$, each in $1 \mathrm{~L}$ of deionised water) and a TYE solution made by adding tryptone and yeast extract to $1 \mathrm{~L}$ deionised water (concentrations of 1\% and 0.5\% respectively). Experimental anodic flow-rates were 1.2, 30, and 240 $\mathrm{mL} / \mathrm{h}$. In between the experiments the flow-rate was maintained at $30 \mathrm{~mL} / \mathrm{h}$. The cathode was continuously hydrated using a second pump (Watson Marlow 205U) that dripped tap water over the electrode ensuring no fluidic connection was possible through the catholyte; the flow-rate was $270 \mathrm{~mL} / \mathrm{h}$ during experiments and kept at $2.4 \mathrm{~mL} / \mathrm{h}$ between experiments and overnight. All experimental runs were repeated at least three times and a similar pattern was always observed. 


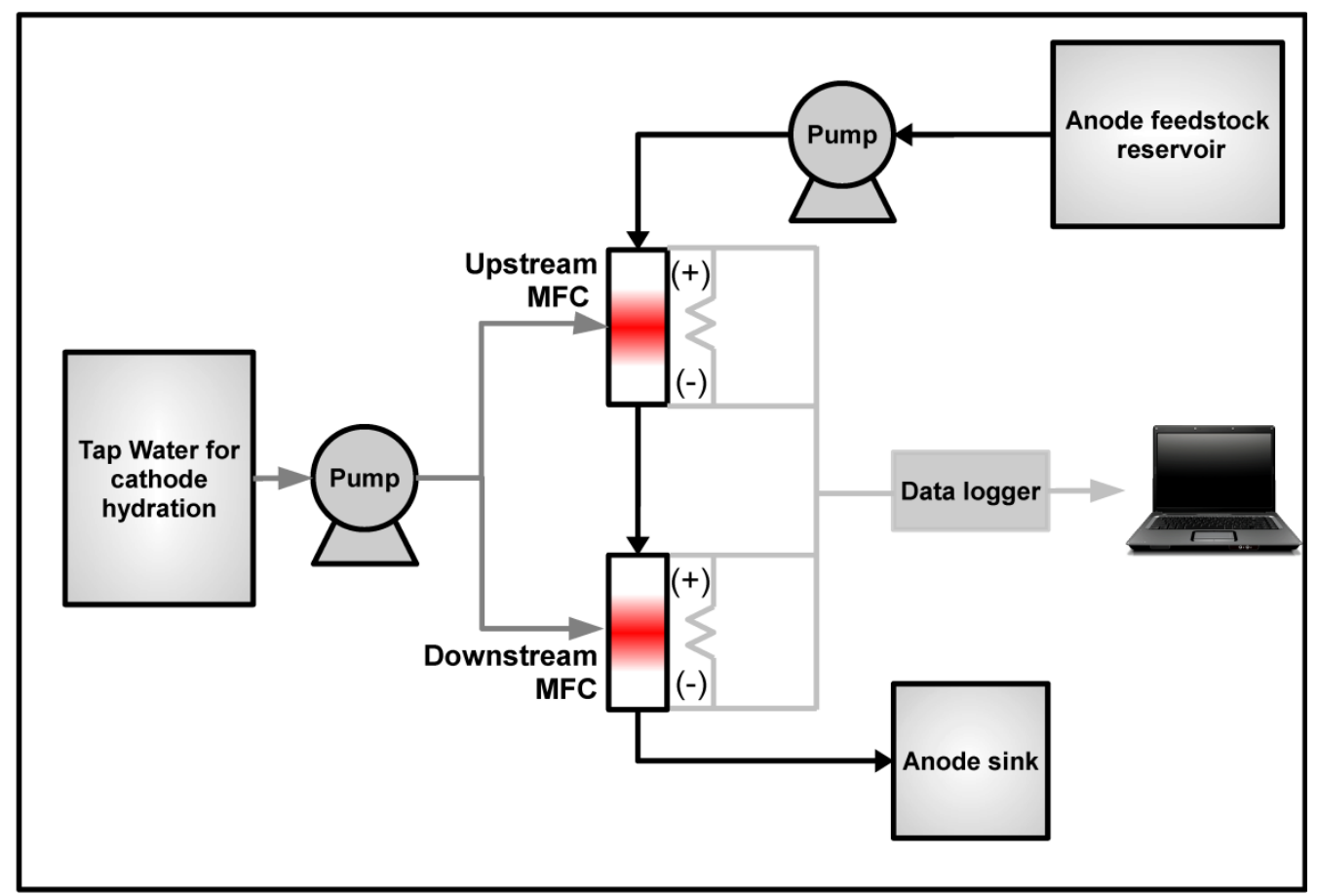

Fig. 2 Schematic representation of two MFCs fluidically joined where feedstock flowed through the anodic chamber of the upstream MFC into the anodic chamber of the downstream MFC

\section{Data capture}

Electrode output was recorded in volts (V) against time using an ADC-16 channel data logger (Pico Technology Ltd., Cambridgeshire, UK). Recorded data were processed and analysed using GraphPad Prism version 4 software package (GraphPad, San Diego, California, USA). Conductivity was measured with a multi-range conductivity metre (HANNA Instruments HI 9033).

\section{Polarisation sweeps}

Polarisation sweeps were performed on MFCup using a variable resistor (Centrad Boite A Decades De Resistances DR07) under varying feedstock and flow-rate conditions and the effect on the OCV of MFCdown was monitored. Data were produced by sweeping resistor values starting at $5 \mathrm{M} \Omega$ and descending to 0 . The time interval between resistance changes was $1 \mathrm{~min}$. All experiments were carried out at room temperature (24 \pm 2 $\left.{ }^{\circ} \mathrm{C}\right)$.

\section{Calculations}

Current (I) was calculated as previously described [12]. Internal resistance (RINT) was calculated using the method described by Ieropoulos et al. [7]:

Equation 1:

$$
\mathrm{R}_{\mathrm{INT}}=\left(\frac{\mathrm{OCV}}{\mathrm{I}_{\mathrm{L}}}\right)-\mathrm{R}_{\mathrm{L}}
$$

where OCV is the open circuit voltage of the MFC, IL is the current under a given load and $\mathrm{RL}_{\mathrm{L}}$ is the given load. 
Voltage drops in the upstream and downstream MFCs were calculated by taking the difference between the lowest value (prior to load removal) and the point $10 \mathrm{~min}$ after the voltage had recovered and stabilised.

\section{Results and discussion}

Effect of a conductive feedstock on fluidically joined MFCs

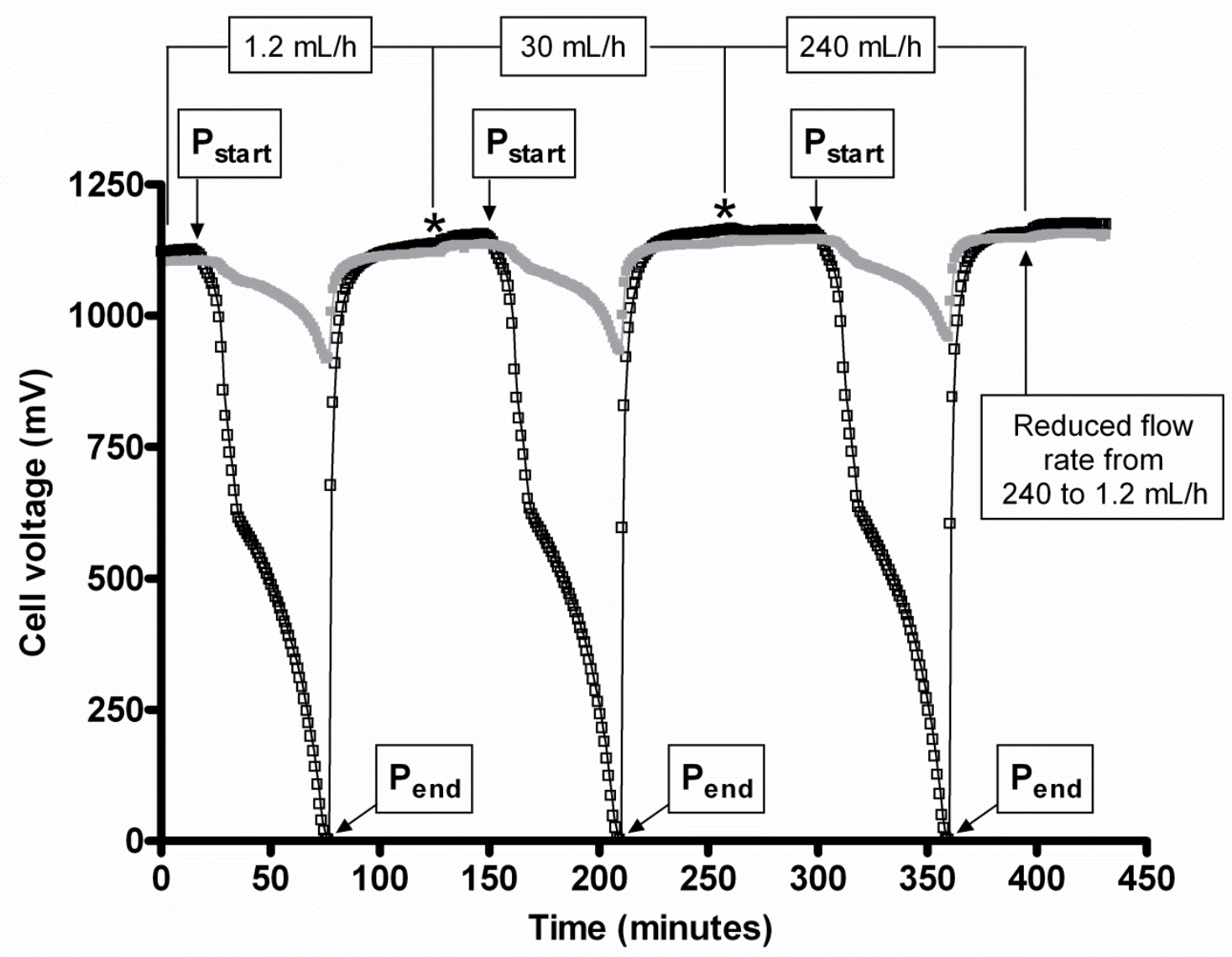

Fig. 3 Voltage behaviour of two fluidically joined MFCs as the upstream MFC (open square) was subject to polarisation sweeps at three different flow-rates; 1.2, 30, and $240 \mathrm{~mL} / \mathrm{h}$. The downstream MFC (closed square) was open circuit, asterisk indicates the point when the flow-rate increased, $P_{\text {start }}$ the start of polarisation sweep and $P$ end the end of polarisation sweep when load removed. TYE solution was used as the feedstock

The highly conductive TYE solution (approximately 4,800 $\mu \mathrm{S}$ ) was used to represent wastewaters in the early stages of treatment (i.e. more enriched). Figure 3 shows the cell voltage of both MFCs as polarisation sweeps were performed on the MFCup at three different flow-rates. The conductive nature of the feedstock resulted in a decline in the MFCdown during the polarisation sweep of MFCup and as the load became heavier the drop was more severe. When the load was removed from MFCup an immediate recovery was observed in MFCdown confirming that the drop was not a result of a depleted substrate. Changes in flow-rate did not directly affect the individual MFCs and had no effect on the magnitude of the maximum voltage drop in MFCdown which was approximately $180 \mathrm{mV}$ in all cases. MFCs were not positionally swapped as part of the current study; subsequent experiments have looked into this aspect and found that the same pattern is observed even when MFC positions are switched, i.e. voltage drop still occurs in the MFCdown (data not shown). Electron leakage can take place between the anode of one MFC and the cathode of another MFC when they are connected both 
hydraulically and electrically in series [10]. During the present study the MFCs were only fluidically connected and so the losses could only have been taking place through the inter-cell feed tube or through the common path of the data logging equipment. To prove that the latter was not the case subsequent experiments were performed with MFCs attached to individual and isolated data loggers and pcs. These experiments showed the same pattern (data not shown). These losses could be in the form of either (1) electronic leakage where electrons travel through the inter-cell feed-tubes linking the MFCs or (2) ionic leakage currents. The latter are known to occur in reverse electrodialysis stacks where ions travel between cells following the development of an electrochemical difference [11]. The losses in MFCdown became greater towards the end of the polarisation sweep when the loads applied upstream were heavier. Decreasing the external resistance will result in a higher number of available electrons flowing through the circuit forcing microbial metabolic activity to increase. This can impact on microenvironmental conditions such as biofilm and membrane $\mathrm{pH}$, ultimately affecting proton transfer [14]. At high current densities these factors become dominant as mass transfer losses. Mass transfer inefficiencies bring about the accumulation of oxidised molecules in the biofilm and can result in sharp increases in the anodic redox potential [15], a factor that will have a detrimental effect on the overall OCV. A method for combatting these concentration losses in conventional fuel cells is the use of small flow channels [16]. The cascade set-up could have been providing one such 'escape' channel where the conductive fluidic connection enabled the accumulated ions to migrate downstream to MFCdown. Such an influx of cations into the anodic chamber of MFCdown could temporarily shift the anodic redox potential to a less negative value, thus decreasing the overall OCV. To examine whether the heavy loads being applied to MFCup were having an immediate effect on the influent that was fed into MFCdown, the conductivity of the feedstock was analysed at varying resistances directly after passing through MFCup. The conductivity remained the same even after being subjected to heavy loads (data not shown). This suggests that the decline in MFCdown was more likely the result of a temporary change in redox potential made possible by the inter-cell connection. Research has shown that individual MFCs when batch-fed perform better at higher conductivities [e.g. 4, 17, 18]. However, as previously stated, stacks of MFCs fed continuously might be a more realistic option when it comes to scaling up. There may, therefore, have to be a trade-off between plurality of units and the conductivity of the surrounding medium they work in. Operating further down in the water treatment process, i.e. as a polishing system [19] might be an environment the cascade could function in without the problems encountered using highly conductive substrates.

\section{Effect of flow-rate on fluidically joined MFCs}

The conductivities of the plain acetate solutions ( $5 \mathrm{mM}$; $320 \mu \mathrm{S}$ and $20 \mathrm{mM}$; 1,100 $\mu \mathrm{S}$ ) were not sufficiently high to act as 'salt-bridges' between the joined MFCs in the same manner as the TYE solution. Figure 4a shows that when fed $5 \mathrm{mM}$ acetate at $1.2 \mathrm{~mL} / \mathrm{h}$ the OCV of MFCdown gently rose and remained stable at $30 \mathrm{~mL} / \mathrm{h}$ with the declining voltage of MFCup having no effect during the polarisation sweeps. The same is witnessed when fed $20 \mathrm{mM}$ acetate (Fig. 4b) where the OCV of MFCdown was very stable at the lower flow-rates. However when the flow-rate increased to $240 \mathrm{~mL} / \mathrm{h}$, MFCdown suffered an instant decline. A drop of $101 \mathrm{mV}$ took place when the 5 $\mathrm{mM}$ acetate media was used and this lower voltage remained stable throughout. An immediate voltage drop is also apparent with the $20 \mathrm{mM}$ acetate feedstock but in this case the OCV continued to decrease reaching a total loss of $223 \mathrm{mV}$. 


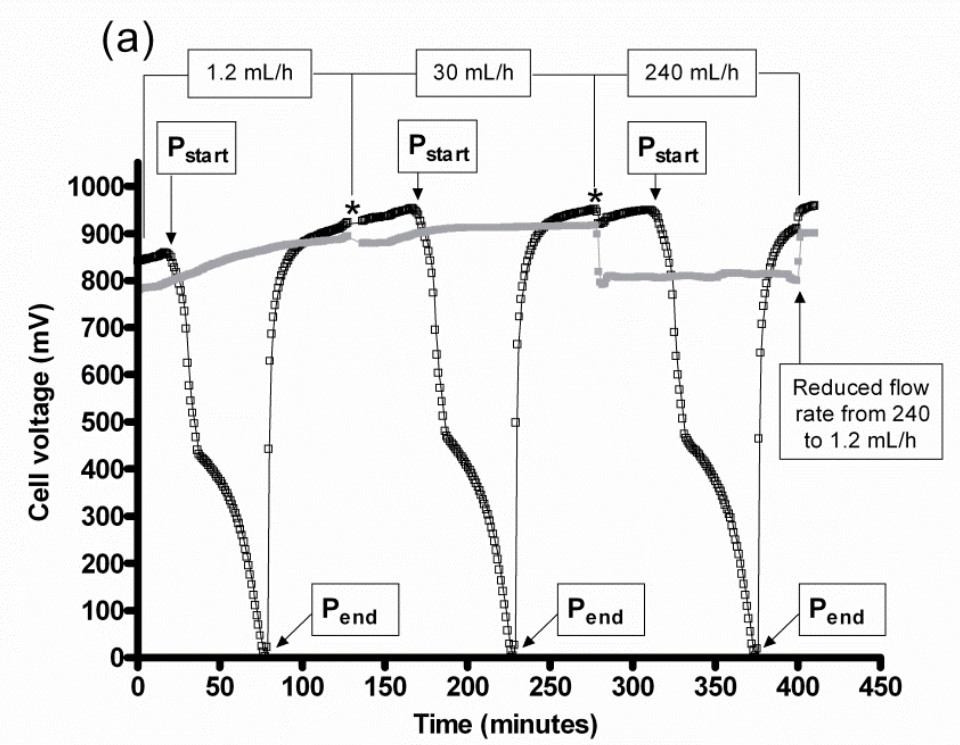

(b)

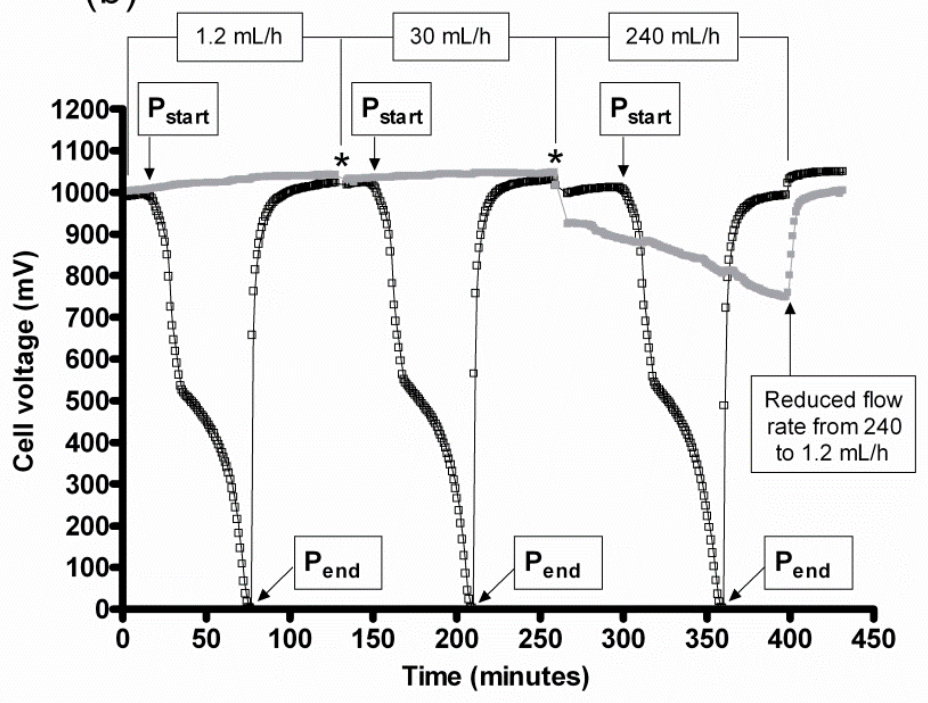

Fig. 4 Voltage behaviour of two fluidically joined MFCs as MFCup (open square) was subject to polarisation sweeps at three different flow-rates; 1.2, 30, and $240 \mathrm{~mL} / \mathrm{h}$. MFCdown (closed square) was open circuit, asterisk indicates the point when the flow-rate increased, $P_{\text {start }}$ the start of polarisation sweep and $P_{\text {end }}$ the end of polarisation sweep when load removed. Feedstock was (a) $5 \mathrm{mM}$ acetate, (b) $20 \mathrm{mM}$ acetate. The distance between MFCs was $20 \mathrm{~cm}$

In both cases immediate recovery occurred when the flow-rate was reduced to $1.2 \mathrm{~mL} / \mathrm{h}$. MFCup was also affected by the increased flow-rate but not as severely ( $49 \mathrm{mV}$ drop at $20 \mathrm{mM}$ and $46 \mathrm{mV}$ at $5 \mathrm{mM}$ acetate). When the distance between the joined MFCs was reduced to $15 \mathrm{~cm}$ these phenomena occurred at lower flow rates than at the larger inter-cell distance. Figure 5 shows that at an inter-cell distance of $15 \mathrm{~cm}$ (when fed 20 $\mathrm{mM}$ acetate) MFCdown remains stable at $1.2 \mathrm{~mL} / \mathrm{h}$ but dropped by $116 \mathrm{mV}$ when the flow-rate was increased to $30 \mathrm{~mL} / \mathrm{h}$ (this flow-rate did not affect the MFCs when the distance was $20 \mathrm{~cm}$ ). MFCup was also affected with a voltage drop of $75 \mathrm{mV}$. These results show that a potentially innocuous substrate might still be a problem through fluidic connections if the conditions are conducive, i.e. short inter-cell distance under high flow-rates. 


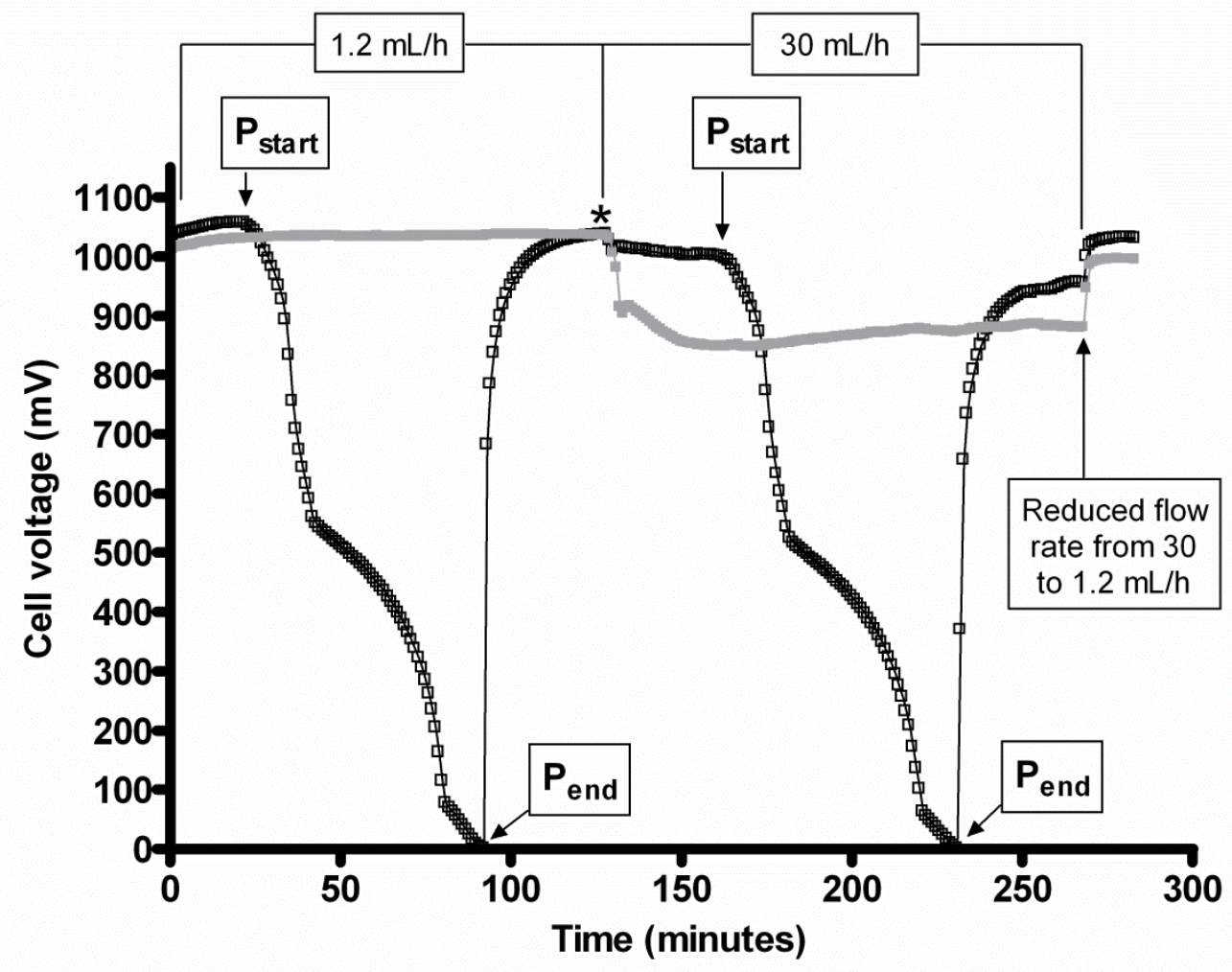

Fig. 5 Voltage behaviour of two fluidically joined MFCs as MFCup (open square) was subject to polarisation sweeps at two different flow-rates; 1.2 and $30 \mathrm{~mL} / \mathrm{h}$. MFCdown (closed square) was open circuit, asterisk indicates the point when the flowrate increased, $P_{\text {start }}$ the start of polarisation sweep and Pend the end of polarisation sweep when load removed. Feedstock was $20 \mathrm{mM}$ acetate. The distance between MFCs was $15 \mathrm{~cm}$

One explanation for this voltage drop might be that the high flow-rate/short-distance combination has introduced a conductive link between MFCs enabling parasitic losses to occur either through electron or ion leakage. However, the diminished OCV in MFCdown remained that way when MFCup was also in open circuit (i.e. before and after the polarisation sweep) suggesting the losses were not the result of current leakage through the fluidic connection but a direct effect of increased flow-rate. The phenomenon of OCV degradation occurs in conventional fuel cells; for example in PEM fuel cells [20] and $\mathrm{PBI}-\mathrm{H}_{3} \mathrm{PO}_{4}$ fuel cells [21]. Further to this, electrochemical reactions can continue in the absence of a load during open circuit conditions in direct methanol fuel cells [22]. This could also be the case for MFCs. The presence of electron acceptors other than the anode electrode (e.g. oxygen) would enable aerobic and facultative species in the anodic community to continue metabolism resulting in substrate depletion but with a loss of electron generation. High flow-rates can introduce increased dissolved oxygen (DO) levels and fluid motion can affect the DO uptake of facultative aerobes [23]. Throughout the experimental period (after inoculation), MFCdown was predominantly in OCV, an environment likely to produce a very different biofilm with alternative metabolic pathways to those selected for closed circuit conditions [24]. In the absence of the anode as electron acceptor, the OCV-microbial community might be more adapted to utilising alternatives such as oxygen. As oxygen diffusion is greater at higher flow-rates the microbial metabolic rate in MFCdown might increase causing further degradation of feedstock, which has been reported to lower the OCV [25]. The same level of metabolic activity could not continue once the flow-rate was lowered and this is reflected by the subsequent rise in OCV. MFCup is not affected as severely as MFCdown probably 
because the biofilm predominantly relied on anodic/anaerobic pathways. High flow-rates can also inflict shearinduced detachment particularly for biofilms grown at low flow-rates [26]. The immediate recovery exhibited by MFCs when the flow-rate was lowered suggests that shear was not a major factor in the present study, however it could be an issue in heterogeneous conditions such as wastewater treatment. The effect that the detachment of microbial cells from one MFC might have on a second MFC especially in terms of a ‘flow-through' anode is an area for future work. Secreted chemicals might also impact on downstream MFCs, e.g. increased shear can induce the short term overproduction of extracellular polymers [27]. Biofilm stability can also be affected by turbulence and the 'flow-through' porous anodes used in the present study may have affected the flow dynamics to MFCdown where even minor variations in flow pattern can affect a receiving biofilm [28]. MFCs operating in wastewater treatment will be exposed to varying degrees of turbulence, shear forces and dissolved oxygen content. Future work will need to examine the effects these may have on long-term performance. When the cascade was fed with the TYE solution there was no detrimental effect seen on MFCdown as a result of flow-rate, but rather as a result of the application of progressively heavier loads. In this scenario the conductive fluidic link was already in place directly joining the two MFCs such that high flow-rates did not affect the equilibrium between the units. It was only the application of loads to MFCup introducing increased electron demand that resulted in a decline in MFCdown. This feedstock was used as a substitute for the types of complex wastewaters found in the early stages of the treatment process where factors such as viscosity can affect oxygen transfer rates [29]. Although not as viscous as aerobic industrial wastes, the complex nature and higher conductivity of the TYE solution might have had an effect on hydrodynamic factors such as the transport and solubility of oxygen.

\section{Internal resistance (RINT) based on a dynamic OCV}

Previously, Ieropoulos et al. [7] showed the importance of the OCV, as an essential parameter for calculating RINT. However, the calculation based on Kirchoff's voltage law uses the starting OCV and makes the assumption that it remains stable throughout, which should be true for all energy generation systems. OCV can degrade over time in some conventional fuel cells and the present study has shown (Figs. 3, 4, 5) that OCV can also be affected by changes in the environmental conditions of MFCs running under load. Therefore, calculating the total RINT based on the starting OCV for a system with potentially a dynamic OCV may result in an erroneous figure. A patent for determining the OCV of a battery in a closed circuit at high current densities is available [13], however, to the authors' best knowledge it is not yet possible to monitor the OCV of a closedcircuit fuel cell at the low current densities produced by MFCs. Laws such as Kirchoff's were defined with conventional batteries and power supplies, and as with all physics laws, they still apply perfectly to these systems. MFCs, however, are reliant on the metabolic behaviour of the resident microbial community, a factor that, particularly in a heterogeneous environment, makes them inherently dynamic and unpredictable. Figure 6 illustrates the OCV and loaded voltage $(30 \mathrm{k} \Omega)$ for MFCdown, when MFCup was undergoing a polarisation experiment. The data in Fig. 6 show that the OCV or loaded voltage of MFCdown was affected by the polarisation run carried out on MFCup. This implies that the OCV equivalent of the actual MFC undergoing polarisation is also changing to at least the same extent. Calculating the total RinT of MFCdown using the starting OCV would, therefore, not be accurate because the OCV changed with time in the same circumstances. 


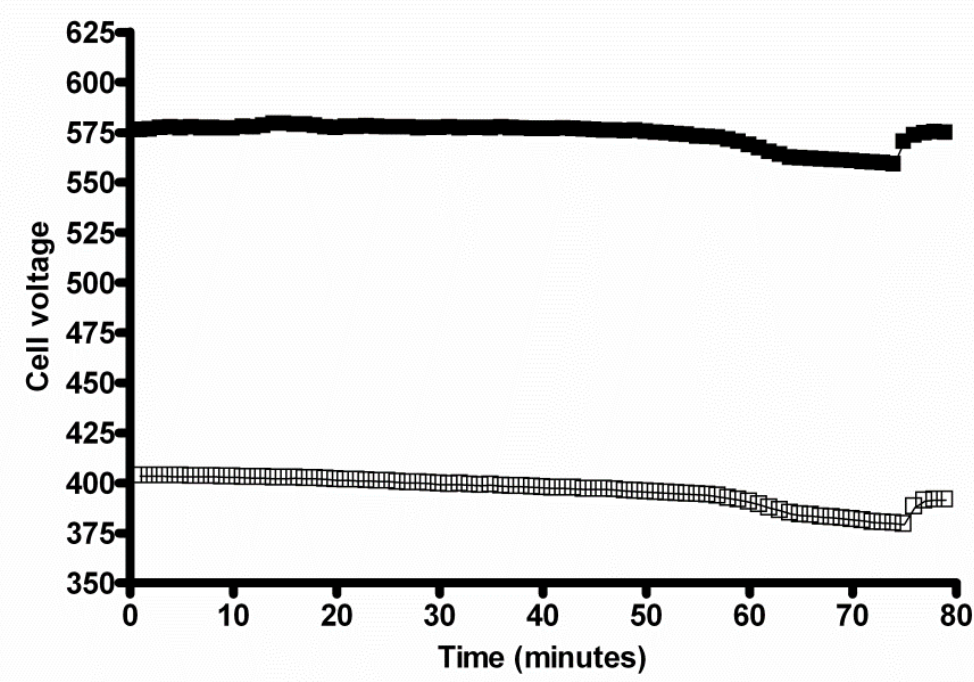

Fig. 6 Behaviour of downstream MFC in open circuit (closed square) and with $30 \mathrm{k} \Omega$ load attached (open square) when fluidically joined to upstream MFC undergoing polarisation (sweep started at 5 mins and ended at 75 mins). TYE solution was used as the feedstock

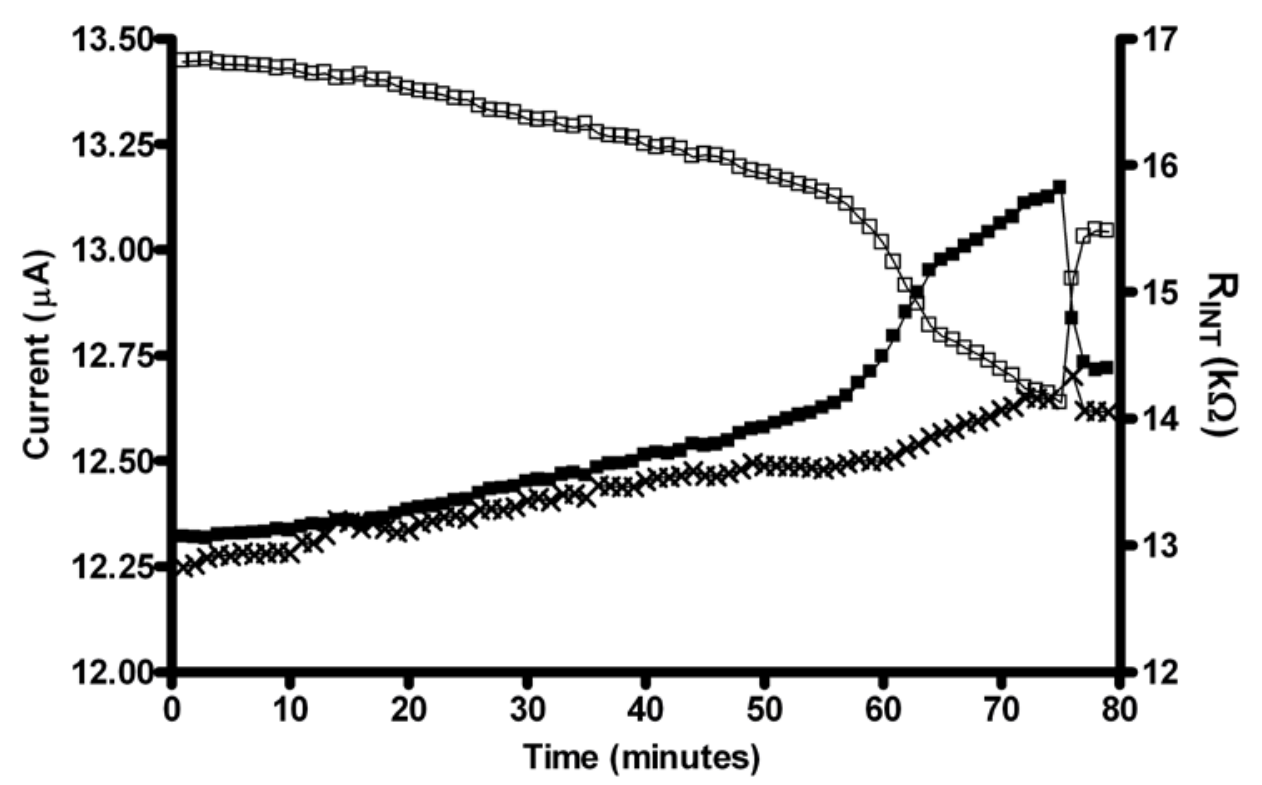

Fig. 7 Comparison of current and RinT in MFCdown when MFCup undergoing polarisation (sweep started at 5 mins and ended at 75 mins). Open square $=$ current, closed square $=$ RINT calculated using static OCV, crosses $=$ RINT calculated using dynamic OCV

Equation 1 was used to calculate RINT for MFCdown during this period of MFCup polarisation based on (1) the static OCV where the starting OCV (579 mV) was used and (2) the dynamic OCV where the changing OCV values were matched to the changing loaded values produced at the same sample time. Calculations based on the static OCV show that the decrease in current was directly reflected by an increase in RINT (Fig. 7). Using the dynamic OCV an increase in RiNT still reflected a decrease in current but the effect was less pronounced and the overall behaviour of RINT was fairly stable. Awareness of a dynamic OCV in bio-electrochemical systems may 
be necessary in future work as it not only governs RiNT but also the maximum power transfer point; a parameter essential for powering target applications.

\section{Conclusions}

These results show that cascades of MFCs operating in a wastewater treatment process will be subjected to losses simply through being fluidically connected. This could be due to the high conductivity of the substrate or high flow rates. These deleterious factors may be avoided by isolating MFCs from one another or by ensuring a significant distance is maintained between units perhaps using a spiralled tubular design. Work is ongoing to explore the potential of using multiples of more than two MFCs in fluidic series, not just in terms of wastewater treatment and power production but as a potential system for signalling changes in organic load and flow-rate.

\section{Acknowledgments}

The authors would like to thank Wessex Water and Great Western Research for their ongoing financial support through GWR project 322.

\section{References}

1. Habermann W, Pommer EH (1991) Biological fuel-cells with sulfide storage capacity. Appl Microbiol Biotechnol 35:128-133

2. Logan BE (2010) Scaling up microbial fuel cells and other bioelectrochemical systems. Appl Microbiol Biotechnol 85:1665-1671

3. Min B, Kim JR, Oh SE, Regan JM, Logan BE (2005) Electricity generation from swine wastewater using microbial fuel cells. Water Res 39:4961-4968

4. $\quad$ Feng Y, Wang X, Logan BE, Lee H (2008) Brewery wastewater treatment using air-cathode microbial fuel cells. Appl Microbiol Biotechnol 78:873-880

5. Greenman J, Galvez A, Giusti L, Ieropoulos I (2009) Electricity from landfill leachate using microbial fuel cells: comparison with a biological aerated filter. Enzyme Microb Technol 44:112-119

6. Shin SH, Choi YJ, Na SH, Jung SH, Kim S (2006) Development of bipolar plate stack type microbial fuel cells. Bull Korean Chem Soc 27:281-285

7. Ieropoulos I, Greenman J, Melhuish C (2008) Microbial fuel cells based on carbon veil electrodes: stack configuration and scalability. Int J Energy Res 32:1228-1240

8. Oh SE, Logan BE (2007) Voltage reversal during microbial fuel cell stack operation. J Power Sources 167:11-17

9. Oh SE, Kim JR, Joo J, Logan BE (2009) Effects of applied voltages and dissolved oxygen on sustained power generation by microbial fuel cells. Water Sci Technol 60:1311-1317

10. Zhuang L, Zhou S (2009) Substrate cross-conduction effect on the performance of serially connected microbial fuel cell stack. Electrochem Commun 11:937-940

11. Veerman J, Post JW, Saakes M, Metz SJ, Harmsen GJ (2008) Reducing power losses caused by ionic shortcut currents in reverse electrodialysis stacks by a validated model. J Memb Sci 310:418-430

12. Ieropoulos I, Winfield J, Greenman J (2010) Effects of flow-rate, inoculum and time on the internal resistance of microbial fuel cells. Bioresour Technol 101:3520-3525

13. Brown WE (1980) Method of determining the open circuit voltage of a battery in a closed circuit. US patent 4204153, US patent and Trademark Office

14. Torres CI, Marcus AK, Rittmann BE (2008) Proton transport inside the biofilm limits electrical current generation by anode respiring bacteria. Biotechnol Bioeng 100:872-881

15. Clauwaert P, Aelterman P, Pham TH, De Schamphelaire L, Carballa M, Rabaey K, Verstraete W (2008) Minimizing losses in bio-electrochemical systems: the road to applications. Appl Microbiol Biotechnol 79:901-913 
16. Wen Q, Wu Y, Cao D, Zhao L, Sun Q (2009) Electricity generation and modeling of microbial fuel cell from continuous beer brewery wastewater. Bioresource Technol 100:4171-4175

17. Liu H, Cheng SA, Logan BE (2005) Power generation in fedbatch microbial fuel cells as a function of ionic strength, temperature, and reactor configuration. Environ Sci Technol 39:5488-5493

18. Huang L, Logan BE (2008) Electricity generation and treatment of paper recycling wastewater using a microbial fuel cell. Appl Microbiol Biotechnol 80:349-355

19. Kim JR, Premier GC, Hawkes FR, Rodriguez J, Dinsdale RM, Guwy AJ (2010) Modular tubular microbial fuel cells for energy recovery during sucrose wastewater treatment at low organic loading rate. Bioresour Technol 101:1190-1198

20. Inaba M, Kinumoto T, Kiriake M, Umebayashi R, Tasaka A, Ogumi Z (2006) Gas crossover and membrane degradation in polymer electrolyte fuel cells. Electrochim Acta 51:5746-5753

21. Qi Z, Buelte S (2006) Effect of open circuit voltage on performance and degradation of high temperature PBI-H3PO4 fuel cells. J Power Sources 161:1126-1132

22. Ye Q, Zhao TS, Yang H, Prabhuram J (2005) Electrochemical reactions in a DMFC under open-circuit conditions. Electrochem Solid-State 8:A52-A54

23. Al-Homoud A, Hondzo M, Lapara T (2007) Fluid dynamics impact on bacterial physiology: biochemical oxygen demand. J Environ Eng-Asce 133:226-236

24. Chung K, Okabe S (2009) Continuous power generation and microbial community structure of the anode biofilms in a three stage microbial fuel cell system. Appl Microbiol Biotechnol 83:965-977

25. Karthikeyan R, Kumar KS, Murugesan M, Berchmans S, Yegnaraman V (2009) Bioelectrocatalysis of Acetobacter aceti and Gluconobacter roseus for current generation. Environ Sci Technol 43:8684-8689

26. Stoodley P, Cargo R, Rupp CJ, Wilson S, Klapper I (2002) Biofilm material properties as related to shear-induced deformation and detachment phenomena. J Ind Microbiol Biotechnol 29:361-367

27. Ramasamy P, Zhang X (2005) Effects of shear stress on the secretion of extracellular polymeric substances in biofilms. Water Sci Technol 52:217-223

28. Gjaltema A, Arts PAM, Vanloosdrecht MCM, Kuenen JG, Heijnen JJ (1994) Heterogeneity of biofilms in rotating annular reactors—occurrence, structure, and consequences. Biotechnol Bioeng 44:194-204

29. Garcia-Ochoa F, Gomez E (2009) Bioreactor scale-up and oxygen transfer rate in microbial processes: an overview. Biotechnol Adv 27:153-176 